\title{
Commentary
}

\section{Risk management in obstetrics: lessons from Australia}

\author{
S Navaneethan ${ }^{1}$ \\ Sri Lanka Journal of Obstetrics and Gynaecology 2009; 31: 10-15
}

Key words: Risk management, litigation, negligence.

\section{Introduction}

To err is human and to learn from error is humane. Unintended outcomes are part of reality in health care. If they result from errors they are considered to be preventable, these outcomes could be improved. Errors are therefore an important starting point to improve patient safety. The concept of risk management in medicine is not new to medicine. Hippocratic oath" states that "I will keep patients from harm and injustice". But this issue became the most popular concept in the recent past because it is seen as a key component in influencing health practitioners' behaviour and possible likelihood of litigation. Obstetrics is a high risk area of practice in Australia as world wide. In obstetrics, litigation can be launched even 25 years after the procedure. Litigation of obstetricians in the recent past had wider implications in healthcare. In the last decade Australia's largest medical defence organisation collapsed due to heavy burden of claims. Currently without government subsidies, obstetricians face annual premiums of up to $\$ 140,000$. A recent survey show there will soon be a shortage of experienced practising obstetricians in Australia².

Idea of this article is to do some brainstorming to work out for a possible risk management strategy for Sri Lanka by analysing the Australian model. Aviation Industry is considered to be the pioneers of advanced risk management process. Aircraft incidents are rare but often involve in massive loss of life, resulting in exhaustive investigations into causal factors, public reports and remedial action. Many lessons are learned and followed in medicine from them. (Interestingly Australian owned Qantas boasts the best flight safety record in flight history with zero fatal events).

Clinical Risk Management (CRM) is an approach to improving the quality and safe delivery of health

${ }^{1}$ Consultant Obstertrician and Gynaecologist, Griffths, Australia.

E-mail:snava5@hotmail.com care by placing special emphasis on identifying circumstances that put patients at risk of harm, and Acting to prevent or control those risks. The Royal College of Obstetricians and Gynaecologists define risk management as:

"methods for the early identification of adverse events, using either staff reports or systematic screening of records; it is an approach to improving the quality of care which places special emphasis on care episodes with unexpected outcomes"

or simply saying not getting things wrong.

\section{Why bother with risk management?}

McNeill and Walton's article ${ }^{3}$ reasons the importance of managing risk, these include. Reducing patient harm and dissatisfaction:

1. Preventing poor use of staff, time and money

2. Protecting from liability and litigation

3. Protecting from bad publicity

4. Making staff aware that there is a spectrum of outcome

5. More explicit and justifiable decision making

6. Improving morale by making organisation more safety conscious

7. Ranking of risks

\section{Components of risk management ${ }^{4}$}

Within a healthcare context risk management has 4 important stages:

1. Risk identification

2. Risk analysis

3. Risk control

4. Risk funding

\section{Risk identification}

Risk identification is the first step in risk management. There are many sources of information are be used in order to understand the risk involved in health care. These include:

\section{a. Clinical Indicators}

Indicators are a measure of the clinical management and outcome of care, objectively 
measuring the process or outcome of care in quantitative terms ${ }^{4}$. Indicators can act as flags, tells where there are problems or issues in patient care. In Australia, a range of organisations collect indicators in obstetrics including Australian Council on Healthcare Standards, State Departments of Health, collecting obstetric and perinatal mortality and morbidity data, National Health and Medical Research Council and Special projects, such as the Maternity Indicators Project in Victoria, Department of Human Services .

\section{b. Incident monitoring}

An incident is "an unplanned event resulting in or having the potential for injury, ill health damage or other loss". Within Australia there are several ways in which incidents are monitored, this happens at both state and federal level. One Commonwealth initiative has been the Australian Incident Monitoring Study (AIMS), funded under the National Hospital Outcomes Program, it was set up to find out not only what, why and how adverse events occur (even near misses), but more importantly to develop improvements to reduce or prevent such events ${ }^{5}$.

Each hospital/area health service have their own system for incident monitoring. The data collected from such incidents is reported to State departments of health, insurance companies and federal government. There a range of methods of collecting this type of data.

\section{c. Adverse or sentinel events}

Australian Quality and Safety Council (2001) define adverse events as "an incident in which harm resulted to a person receiving health care" ${ }^{115}$ Serious events such as death or serious physical or psychological injury are stated as sentinel. These are rare but significant events that warrant individual investigation and a detailed response. Information about adverse events is collected and reported to a range of sources, if it has involved a death it will be reported to the coroner.

\section{d. Limited outcome occurrence screening}

Adverse events are also identified by doing a retrospective search of hospital-based information, using selection criteria. Using this method helps in identifying past events that staff may be unaware of, and requires dedicated time and administrative support.

\section{e. Patient complaints}

Patient complaints often arise from areas inappropriately managed. The way in which patient complaints are dealt with, by taking them seriously, responding quickly and sensitively may help solve things earlier preventing them from escalating into legal action.

\section{Risk analysis}

Analysing several sources of data provide a rich source of information that is used in the analysis phase. Incidents are analysed in order to establish how often they happen and what their effects might be. This help to concentrate and focus attention on getting these aspects of care right. This analysis on a grand scale is used hospital or state level to inform strategies and initiatives across services.

\section{Root cause analysis}

Root cause analysis (RCA) is a methodical way of analysing what goes wrong in order to prevent them happening again. There is a range of tools that are used within the context of carrying out a root cause analysis.

\section{Risk Control}

Once the risks have been identified and analysed, it is considered how they can be avoided, reduced, transferred or eliminated. This can be through physical and systems controls, but also requires an understanding of how doctors interact with patients and members of the multidisciplinary team.

Technical and human errors play a large part in incidents and adverse events, and can often be pinpointed so that they can be controlled. Some risk can never be completely eliminated, but likelihood of it arising could be reduced.

There are four ways of coping with risk: avoid, reduce, transfer and retain.

Risk Avoidance: Not proceeding with an activity that poses an unacceptable risk. An example of this is where an O\&G drops their obstetrics work.

Risk Reduction: Choosing an alternative course with less risk: An example of this may be choosing Vacuum to reduce vaginal tears arising from Forceps delivery.

Risk Transfer: For example transfer of severe Heart disease complicating pregnancy patient to a tertiary unit

Risk Retainment: To retain a risk even though there are other methods of handling the risk. As an example, a hospital may decide for political and social reasons 
that they will still provide some services to high-risk patients rather than transferring them to a tertiary centre.

\section{Communication}

Communication and attitude are aspects of health care delivery are critical in influencing patient relationships and their propensity to complain and even litigate. Majority of claims made in Australia are against doctors can be traced back to communication problems ${ }^{7}$.

\section{Team issues}

Operating within a team environment makes staff communication errors. Recognising that communication and stress within the team can lead to mistakes is important and can be improved through changing the hierarchical nature of the work environment and the ways staff interact.

\section{System factors}

The processes and way we organise health care are considered to be 'systems'. The ways in which systems operate or fail abysmally are often the factors that lead to adverse events. This can range from equipment failure to protocols not being followed.

\section{Equipment failure}

Checking and maintaining tools and equipment of procedures is often overlooked, or there is no schedule for regular servicing ${ }^{7}$.

\section{Guidelines, protocols and standards}

One common factor mentioned during litigation against doctors is a failure to follow the guidelines or local protocols. Guidelines are often cited as standard or accepted practice ${ }^{8}$.

\section{Documentation}

Poor quality of documentation is an oft-cited reason why many doctors and MDO's (Medical defence organizations) are unable to defend claims. Providing clear, legible and understandable documentation covers people involved when things go wrong and helps others understand what happened during a patient episode. Relying in memory may be inadequate, especially recalling the details of a case that happened.

\section{Risk funding}

Professionals and the hospitals consider how the residual risk is paid for and the available money is best spent.

\section{Practical model of incident management process ${ }^{9}$}

Different models are practiced Australia wide but the concepts are the same. The seven key steps to effective incident management are: identification, notification, prioritisation, investigation, classification, analysis and action and feedback.

\section{Step 1 - Identification}

Staff recognises an incident. Following identification of an incident or near miss there may need to be immediate action. These actions may include:

1. Providing immediate care to individuals involved in the event (patient, staff or visitors)

2. Making the situation/scene safe to prevent immediate recurrence of the event

3. Removing malfunctioning equipment or supplies

4. Gathering basic information about a chain of evidence

5. Notifying police and security

\section{Step 2 - Notification}

Staff are required to notify all identified incidents, near misses and complaints in the Incident Information Management System(IIMS). In Australia most of the health services have computerised information system in which health staff make entries whenever they encounter incident. This system ensures and promotes all incidents are dealt appropriately.

2.1 Documentation of the incident in the health record

2.2 Incident notification in IIMS - by the notifier

This must occur as soon as possible, can be identified or anonymous, apart from workplace injury notification. The notifier is asked to undertake an initial assessment of severity of the incident using the Severity Assessment Code (SAC) Matrix and give their opinion of how the incident may have been prevented. The patient and/or their family or carer can notify an incident through the complaints management process in place in each health service.

2.3 Incident notification - management responsibility The manager reviews the IIMS notification, completes the IIMS management screen, and either allocates or confirms the SAC score according to the actual incident or near miss. 
2.4 Notification to patient (open disclosure)

All SAC 1 and 2 events (Those with serious outcome, such as patient death, severe bodily harm) are to be accompanied by the full open disclosure process. The initial disclosure to the patient and / or their support person must occur within 24 hours of the incident, by the health care professional responsible for the care of the patient. When an actual clinical incident occurs to a patient, an integral component of the notification process is to acknowledge the occurrence of the incident to the patient and their support person, as appropriate and to inform them of the type of investigation that will be undertaken an apology for the incident suffered is given at this stage.

\section{Step 3 - Prioritisation}

The purpose of prioritisation is to ensure that a standardised, objective measure of severity is allocated to each incident or near miss. This enables an appropriate level of investigation to be conducted. The Severity Assessment Code (SAC) is used to prioritise all notifications. The SAC is a matrix that takes into account both the consequences of the incident (or near miss), and the likelihood of recurrence of the incident (or near miss) to apply a numerical rating. The SAC score guides the level of investigation and the need for additional notification. All Severity Assessment Code (SAC) 1 incidents are escalated to the Chief Executive of the organisation.

\section{Step 4 - Investigation}

Investigation of the incident is an important component of any patient safety program. All incidents notified in IIMS require an investigation.

All clinical SAC 1 incidents must have the final RCA report completed and submitted to the Department within 70 calendar days from the notification of the incident in IIMS $^{10}$. All corporate SAC 1 Incidents must have a detailed investigation completed and a report submitted to the Department within 70 calendar days from the notification of the incident in IIMS.

\section{Levels of investigation}

All notified incidents require a review to assess the level of investigation required. The SAC score guides the level of investigation.

\section{SAC 1 Incidents}

All clinical SAC 1 incidents require a privileged Root Cause Analysis (RCA) investigation. The final report of the SAC 1 incident investigation should be presented to the Clinical Team involved in care of the patient and at relevant staff meetings to ensure all staff are aware of the factors contributing to the incident and the action being taken to improve safety.

\section{SAC 2 Incidents}

Require a detailed investigation of the incident. Ongoing monitoring of trended aggregated incident data may also identify and prioritise issues requiring a practice improvement project. The responsibility assigned to a designated senior manager and Investigation should be completed within 28 days as monitored in IIMS or a progress report outlining the management plan with a revised completion date being submitted to the appropriate senior manager.

\section{SAC 3/4 Incidents}

The investigation of SAC 3 and 4 incidents can be undertaken at the local level but management responsibility for the investigation must be assigned. Any financial loss must be reported to senior management. Investigation of SAC 3/4 incidents may involve, interviews with staff and patient and/or family involved, review of policies/procedures, record review, clinical/performance indicators or other relevant material. Investigation should be completed, where possible, within 28 days as monitored in IIMS or a progress report outlining the management plan with a revised completion date being submitted to the appropriate senior manager.

An aggregated identified report on all corporate SAC 1, 2, 3 \& 4 incidents is to be provided to the health service's Audit and Risk Management Committee. An aggregated report on all OH\&S incidents is to be provided to the Director, Workforce Development and any relevant $\mathrm{OH} \& \mathrm{~S}$ Committee.

\section{Investigations and individual performance}

Investigations conducted under this policy do not canvass issues of individual performance. Where a question of individual performance arises, this is managed via the organisation's performance management system.

\section{Director General inquiries under the Health Services Act}

A limited number of clinical incidents can raise issues which may require a more formal inquiry to review a matter independently of the public health organisation. 
This may arise where a clinical incident raises broad state wide or general clinical practice issues, serious public interest matters or matters where there is a potential conflict of interest in the organisation overseeing an investigation itself. Where the CE considers an independent external inquiry may be required, they contact the $\mathrm{DOH}$ Legal Branch and the DOH Corporate Governance and Risk Management Branch.

\section{Step 5 - Classification}

This is the process of capturing relevant information from a range of perspectives about an incident to ensure that the complete nature of the incident, including causative and contributory factors, is documented and understood. Classification of all incidents involving patients, staff, visitors, volunteers, contractors or corporate.

\section{Step 6 - Analysis and action}

The purpose of analysis is to understand how and why the incident occurred, and to identify ways of preventing a recurrence. The analysis should take into account all information gathered during the Investigation and Classification phases. Actions and recommendations are developed to prevent recurrence of the incident. A suitable timeframe for the implementation of recommendations is documented in IIMS. Senior management is responsible for deciding whether recommendations are accepted and approved and for appropriate resource allocation. A senior manager records the acceptance of recommendations and/or comments in IIMS once the recommendations have been approved by the $\mathrm{CE}$, The IIMS system is used to capture actions and recommendations, and to flag follow up and review dates. Ongoing monitoring is required to ensure recommendations are addressed in a timely manner and to evaluate the success of any action taken to achieve improvement.

\section{Step 7 - Feedback following investigation}

Feedback is an important component of a successful incident management programme.

\section{Feedback to Patients and/or Support Person (Open Disclosure)}

Information about SAC 1 and SAC 2 clinical incidents offered to the patient and / or their support person. The information provided to the patient and / or their support person can be based on a variety of sources. The final report from a privileged RCA is one of the sources that may be used in providing feedback on a clinical SAC 1 incident.

\section{Feedback to Staff}

The success of incident management is dependent on feedback to all staff on the results/ outcomes of investigations in a timely manner. Staff involved in the incident need to be informed of the recommendations arising from any investigation. The final RCA Report provides the basis for feedback on a clinical SAC 1 clinical incident. The findings of the Clinical SAC 1 RCA Report should be provided to the relevant clinical team and presented at relevant staff meetings. Feedback needs to include the changes made and improvements achieved as a result of these changes.

\section{Reportable Incident Briefs}

The Reportable Incident Brief (RIB) system is designed for the reporting of specific health care incidents to the NSW Department of Health. The RIB process encompasses clinical and corporate incidents.

Clinical incidents: all clinical incidents reported in RIBs are referred to the NSW Health Reportable Incident Review Committee (RIRC). The Committee is responsible for examining and monitoring serious clinical adverse events collected by the Committee in Reportable Incident Briefs (RIBs). The Committee analyses information and identifies issues relating to morbidity and mortality that may have State wide implications and provides advice on policy development to effect health care system improvement.

\section{$R I B$ reporting requirements}

All actual SAC 1 incidents, both clinical and corporate, are required to be notified to the Department, via a RIB, within 24 hours of notification in IIMS.

\section{Sri Lankan situation}

Until recently litigation in medical practice hasn't been a burning issue in Sri Lanka. But this will change and this safe haven may not last longer. Sri Lanka claims one of the best rates in MMR, but we shouldn't rest on our laurels. It's essential that we should look forward in working towards a conducive risk management plan to further improve our state of maternal health.

In Sri Lanka we have to confess that our risk identifying sources are quite limited and poorly developed. Risk identification is the first step in risk management. We do have the statistics through the Health Department and MMR analysis through the College, but unfortunately they are under reported and poorly analysed to yield any productive changes. Maternal morbidity and mortality meetings are often 
places to justify the management of the particular patients rather than deploring the causes and future preventions. Regular reporting and review of incidents in books is equally effective to a computerized system, if followed honestly. It is important a proper root cause analysis (RCA) system is developed to identify the causes of incidents. Expert reviews in each incident cannot be over emphasized. We rarely see any changes even any recommendations made, person found guilt will continue business as usual.

MMR in Australia is low and one of the best and stable for the last 20 years in the world. Australia is still striving to further improve the rates. The RANZCOG plays a key role in educating and practicing risk management in Obstetrics. Many projects including AROMA are introduced and functioning effectively. College monitors the Obstetricians participation in risk management activity through mandatory point requirements for Risk management activities in their CPD programmes. As the equal body SLCOG needs to take the vital role of raising the awareness of concept of risk management among the Obstetricians to promote safe motherhood.

\section{References}

1. Hippocratic Oath.

2. Mac Lennan AH, Spencer MK. Projections of Australian obstetricians ceasing practice and the reasons. MJA 2002; 176(9): 425-8.

3. O'Rourke A. Clinical Risk Management, Wisdom Centre, Institute of General Practice \& Primary Care, Sheffield University 2003.

4. AROMA Project. Royal Australian and New Zealand College of Obstetricians and Gynaecologists.

5. Department of Health and Ageing. Incident monitoring improving patient safety. Better Health Outcomes newsletter, 1997: 3(1).

6. Gorney M, Bristow J. Effective physician communication skills. The Doctors Company 2002.

7. Pelly JE, et al. Clinical practice guidelines before the law: sword or shield? MJA 1998; 169: 330-3.

8. Health Policies. Department of Health. NSW 